\title{
Biochemistry and genetics of eukaryotic mismatch repair
}

\author{
Richard Kolodner \\ Charles A. Dana Division of Human Cancer Genetics, Dana-Farber Cancer Institute, Boston, Massachusetts 02115 USA and \\ Department of Biological Chemistry and Molecular Pharmacology, Harvard Medical School, Boston, Massachusetts 02115 \\ USA
}

The process of mismatch repair was first postulated to explain the results of experiments on genetic recombination and bacterial mutagenesis. Mismatch repair has long been known to play a major role in two cellular processes: (1) the repair of errors made during DNA replication or as the result of some types of chemical damage to DNA and DNA precursors; and (2) the processing of recombination intermediates to yield new configurations of genetic markers. More recent studies have suggested that mismatch repair may also be crucial for (1) the regulation of recombination events between divergent DNA sequences that could result in different types of genetic instability (Rayssiguier et al. 1989; Selva et al. 1995; Datta et al. 1996), (2) some types of nucleotide excision repair responsible for repair of physical/chemical damage to DNA (Karran and Marinus 1982; Fram et al. 1985; Feng et al. 1991; Mellon and Champe 1996), and (3) participating in a cell-cycle checkpoint control system by recognizing certain types of DNA damage and triggering cell-cycle arrest or other responses to DNA damage (Hawn et al. 1995; Anthoney et al. 1996).

The most extensively characterized general mismatch repair system is the Escherichia coli MutHLS system, which repairs a broad spectrum of mispaired bases and has been reconstituted with purified enzymes. Eukaryotes are known to contain a mismatch repair system that has at least some components that are highly related to key components of the bacterial MutHLS mismatch repair system. The observation that defects in mismatch repair genes are linked to both inherited cancer susceptibility and some sporadic cancers has generated considerable interest in the gene products that function in eukaryotic mismatch repair. The goal of this review is to discuss recent studies on the mechanisms of MutHLSlike mismatch repair in the yeast Saccharomyces cerevisiae and in humans and to relate insights derived from these studies to human cancer genetics. Given space constraints, it is difficult to cover everything known about mismatch repair or to reference all of the relevant work that has been done in this area. However, a brief overview of the E. coli MutHLS pathway is presented below to allow comparison of the $E$. coli and eukaryotic mismatch repair pathways and proteins. For more detailed information, particularly related to bacterial mis- match repair, base-specific mismatch repair systems, and cancer genetics, see other recent reviews (Modrich 1991; Eshleman and Markowitz 1995; Fishel and Kolodner 1995; Friedberg et al. 1995; Kolodner 1995; Marra and Boland 1995; Modrich and Lahue 1996).

\section{The $E$. coli MutHLS mismatch repair pathway}

The E. coli MutHLS mismatch repair pathway is a general DNA repair pathway that recognizes and repairs all single-base mispairs except C.C (Modrich 1991; Fishel and Kolodner 1995; Friedberg et al. 1995; Kolodner 1995; Modrich and Lahue 1996). It also repairs small insertion/ deletion mispairs, although it may not efficiently recognize most insertion/deletion mispairs that have more than 4 unpaired bases. The basic repair reaction catalyzed by this pathway is understood in considerable detail because it has been reconstituted in vitro with DNA substrates containing mispaired bases, MutH, MutL, MutS, and UvrD (helicase II) proteins, DNA polymerase III holoenzyme, DNA ligase, single-strand DNA-binding protein $(S S B)$, and any one of the single-stranded DNA exonucleases-Exo I, Exo VII, and RecJ protein (Fig. 1; Lahue et al. 1989; Modrich 1991; Grilley et al. 1993; Fishel and Kolodner 1995; Friedberg et al. 1995; Kolodner 1995; Modrich and Lahue 1996). The reaction involves mismatch-dependent nicking of the unmethylated strand at a hemimethylated GATC site and degradation from the nick past the mismatch followed by resynthesis. The roles of many of the proteins that function in this reaction have been elucidated. The MutS protein binds to DNA at the site of a mispaired base and is responsible for mismatch recognition. No activity has been assigned to MutL, although it interacts with MutS bound to a mispaired base and is required for activation of MutH. MutH is an endonuclease that nicks hemimethylated DNA on the unmethylated strand when activated by MutS and MutL in the presence of a mismatch. The requirement for $\mathrm{MutH}$ in the reaction can be replaced by a pre-existing nick in the DNA. The repair reaction can utilize hemimethylated sites that are either $5^{\prime}$ or $3^{\prime}$ to the mispair. Excision requires UvrD (helicase II) and one of the single-stranded DNA exonucleasesExo I $\left\{3^{\prime}\right.$ exonuclease $)$, Exo VII $\left\{3^{\prime}\right.$ and $5^{\prime}$ exonuclease $)$, 


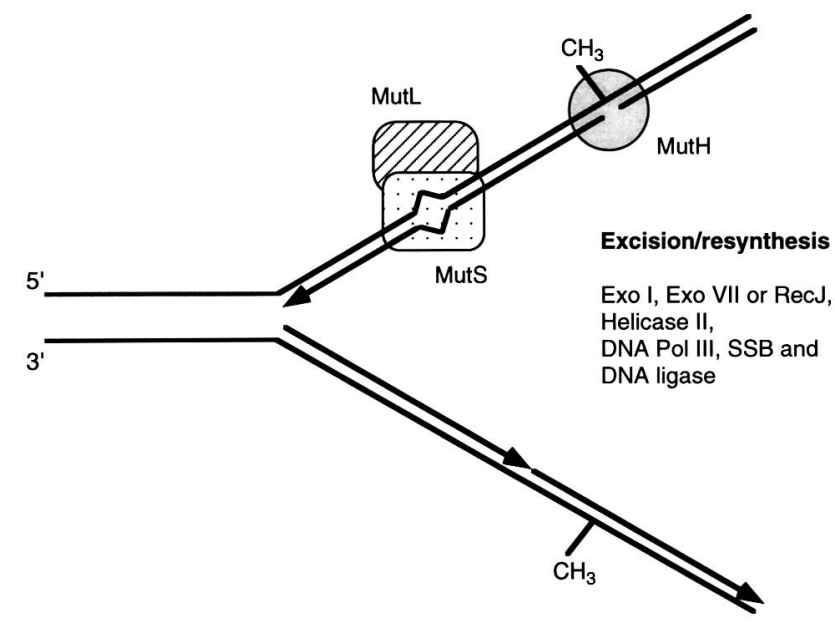

Figure 1. Illustration of the action of the E. coli MutHLS mismatch repair system on a mispair at a replication fork. Repair is initiated by binding of MutS protein to a mismatch. The subsequent binding of MutL to MutS is required to activate MutH, which then nicks the unmethylated strand of DNA at hemimethylated GATC sites. Nicking of the unmethylated strand is then followed by the excision from the nick to the mispair and resynthesis to fill in the resulting gap. These interactions result in the coupling of mismatch repair to DNA replication, so that mismatches formed during DNA replication are repaired using the methylated parental strand as template, resulting in a reduction of misincorporation errors. (Adapted from Kolodner 1995.)

and RecJ protein $15^{\prime}$ exonuclease $/$-depending on whether the nicked, unmethylated site is $5^{\prime}$ or $3^{\prime}$ to the mispair. Once excision has occurred, resynthesis is mediated by DNA polymerase III holoenzyme, SSB, and DNA ligase.

A crucial feature of the MutHLS mismatch repair pathway is its ability to preferentially repair the daughter DNA strand after DNA synthesis, thus increasing the fidelity of DNA replication. Normally, DNA in E. coli is methylated at GATC sites by the Dam methylase. This is a postreplication modification, however, and the daughter DNA strand is transiently unmethylated after DNA replication. The MutHLS system utilizes this modification asymmetry to direct repair to the unmethylated daughter strand via the activity of $\mathrm{MutH}$, which nicks the unmethylated DNA strand at hemimethylated GATC sites. It is important to point out that the requirement for MutH and the use of Dam methylation to distinguish between newly replicated and parental DNA appear to be unique to certain bacteria. Other bacteria like Streptococcus pneumoniae have a mismatch repair system that is closely related to the MutHLS system but the Streptococcus system, referred to as the Hex system, is not presently known to utilize a MutH homolog or DNA methylation as a mechanism for strand discrimination in the repair process (Modrich 1991; Fishel and Kolodner 1995; Friedberg et al. 1995; Kolodner 1995; Modrich and Lahue 1996). DNA methylation does not occur in S. cerevisiae (Proffitt et al. 1984), and no con- vincing evidence has implicated DNA methylation in strand discrimination during mismatch repair in other eukaryotes (Modrich 1991; Fishel and Kolodner 1995; Friedberg et al. 1995; Kolodner 1995; Modrich and Lahue 1996). Thus, it seems possible that the mechanism of strand discrimination in eukaryotes will prove to be quite different from that observed in E. coli.

\section{MutHLS-like mismatch repair in eukaryotes}

Two lines of experimentation have indicated that eukaryotes have a broad-spectrum mismatch repair system related to the bacterial MutHLS system. First, a series of genetic studies led to the identification of $S$. cerevisiae homologs of the bacterial mutL and mutS gene products and the demonstration that these gene products were required for mismatch repair (Williamson et al. 1985; Bishop et al. 1987; W. Kramer et al. 1989; Reenan and Kolodner 1992a; Reenan and Kolodner 1992b; Kolodner 1995). Second, biochemical studies of higher eukaryotic cells have demonstrated in vitro nick-directed repair of a variety of different mispaired bases similar to the repair reactions catalyzed by the $E$. coli MutHLS system (Fang and Modrich 1993; Modrich and Lahue 1996). More recently, biochemical studies have demonstrated that these mismatch repair reactions are dependent on eukaryotic homologs of the bacterial mutL and mutS gene products (Umar et al. 1994; Boyer et al. 1995; Drummond et al. 1995; Li and Modrich 1995). A considerable body of evidence indicates that this pathway plays an important role in maintaining replication fidelity, processing recombination intermediates, and regulating recombination in response to sequence divergence. These subjects, however, are beyond the scope of this review.

\section{Homologs of the bacterial MutS proteins}

A series of studies has demonstrated that there are at least six S. cerevisiae proteins, MSH1-MSH6, which show a high degree of amino acid similarity with the Bacterial MutS proteins (Fig. 1; Table 1). Homologs of many of these proteins have been identified in other organisms and are the subject of intense study. As will be discussed below, there is evidence that three of these proteins, MSH2, MSH3, and MSH6, function in a eukaryotic MutHLS-like mismatch repair pathway. The involvement of three MutS homologs in mismatch repair provides evidence for the relative complexity of eukaryotic mismatch repair compared to the bacterial paradigm.

It was originally thought that the eukaryotic MSH2 protein, a homolog of the bacterial MutS protein, was the mismatch repair protein that recognizes mispaired bases in DNA (Reenan and Kolodner 1992a,b). Purified MSH2 from both $S$. cerevisiae and human cells recognizes both single-base mispairs and, with a higher affinity, multiple base insertion/deletion mispairs (Fishel et al. 1994; Alani et al. 1995). More recently, it has been appreciaced that human MSH2 copurifies with a second MutS homolog, human GTBP/p160 (Drummond et al. 1995; Pal- 
Table 1. Names and alternate names for mismatch repair genes and proteins discussed

\begin{tabular}{lll}
\hline E. coli protein & S. cerevisiae protein & Human protein \\
\hline MutS & MSH2 & MSH2 \\
& MSH3 & MSH3, DUG1, MRP1 \\
MutL & MSH6 & GTBP, p160 \\
& PMS1 & PMS2 \\
& MLH1 & MLH1 \\
& MLH2 & PMS1 \\
\hline
\end{tabular}

General nomenclature: Gene names, uppercase italics; mutation names, lowercase italics; protein names, uppercase except for E. coli poteins, where only the first letter of each word is capitalized.

Note on MutL-related proteins: Human PMS2 is more closely related to $S$. cerevisiae PMS1 than human PMS1 is related to $S$. cerevisiae PMS1. Human PMS1 is more closely related to $S$. cerevisiae MLH2 (see Fig. 4).

References: E. coli MutS-related proteins: (Reenan and Kolodner 1992a, Fishel et al. 1993; Leach et al. 1993; New et al. 1993; Drummond et al. 1995; Palombo et al. 1995; Marsischky et al. 1996; Watanabe et al. 1996). E. coli MutL-related proteins: (Bronner et al. 1994; Nicolaides et al. 1994; Papadopoulos et al. 1994; Prolla 1994a,b).

ombo et al. 19951, which appears to be the homolog of $S$. cerevisiae MSH6 (Figs. 2 and 3, and below). The observation that the heterodimer of human MSH2 and GTBP (MSH6) recognizes mispaired bases initially suggested that the entire complex could function as the MutS equivalent in eukaryotic mismatch repair (Drummond et al. 1995; Palombo et al. 1995). The available data on mismatch repair-defective tumor cell lines, however, suggest that the role of a MSH2-GTBP (MSH6) complex in mismatch recognition may not be simple. In vivo, tumor cell lines lacking MSH2 appear to have a high degree of microsatellite instability at both dinucleotide and mononucleotide repeat loci (Bhattacharyya et al. 1994; Shibata et al. 1994; Umar et al. 1994b; Boyer et al. 1995; Papadopoulos et al. 1995). In contrast, tumor cell lines lacking GTBP (MSH6) appear to have less pronounced microsatellite instability: Dinucleotide repeat instability is difficult to detect in such cell lines, and mononucleotide repeat instability is less pronounced compared to msh2 mutant lines (Bhattacharyya et al. 1994; Shibata et al. 1994; Papadopoulos et al. 1995). Biochemical analysis also suggests that mutations in $\mathrm{MSH} 2$ and GTBP (MSH6) have somewhat different effects on mismatch repair in in vitro mismatch repair assays (Drummond et al. 1995). Finally, mutations in MSH2 are common in hereditary nonpolyposis colorectal cancer (HNPCC) families, whereas mutations in GTBP (MSH6) have not been found (Papadopoulos et al. 1995; Liu et al. 1996). These data suggest that MSH2 and GTBP (MSH6) are not genetically equivalent.

Genetic and biochemical analyses of mismatch repair in $S$. cerevisiae have suggested that mismatch recognition involves three MutS homologs: $\mathrm{MSH} 2, \mathrm{MSH} 3$, and MSH6 (the homolog of human GTBP/p160), which form two different heterodimeric complexes (Marsischky et al. 1996). Mutations in MSH2 were found to cause a strong general mutator phenotype resulting in the accumulation of both frameshift and single-base substitution mutations, high rates of frameshift mutation reversion, and dinucleotide repeat instability, consistent with a general role in mismatch repair (Reenan and Kolodner 1992a; Strand et al. 1993; Marsischky et al. 1996). In contrast, mutations in MSH6 caused only a modest mutator phenotype that was confined primarily to the accumulation of single-base substitution mutations, suggesting that MSH6 might be required primarily for recognition of single-base substitution mispairs and to a lesser extent single base insertion/deletion mispairs (Marsischky et al. 1996). Consistent with this latter view, pms3 mutants, which had been shown previously in transformation assays that detect mismatch repair directly to cause a defect in the repair of single-base substitution mispairs but not in the repair of single-base insertion/deletion mispairs, are caused by mutations in MSH6 (B. Kramer et al. 1989; Marsischky et al. 1996). Also consistent with this view is the observation that msh6 mutations cause only a small increase in dinucleotide repeat instability (Johnson et al. 1996).

Unlike either msh2 or msh 6 mutants, msh3 mutants have little if any mutator phenotype in forward mutation and frameshift reversion assays and show increased dinucleotide repeat instability, albeit at lower levels, than that seen in msh2 mutants (New et al. 1993; Alani et al. 1994; Strand et al. 1995; Marsischky et al. 1996;). Strikingly, when double mutant strains were analyzed, msh3 and msh 6 mutations showed a large synergistic effect on the rate of accumulation of mutations in frameshift reversion assays that measure the formation and repair of single-base insertion/deletion mispairs (Marsischky et al. 1996). A similar synergistic effect was seen in forward

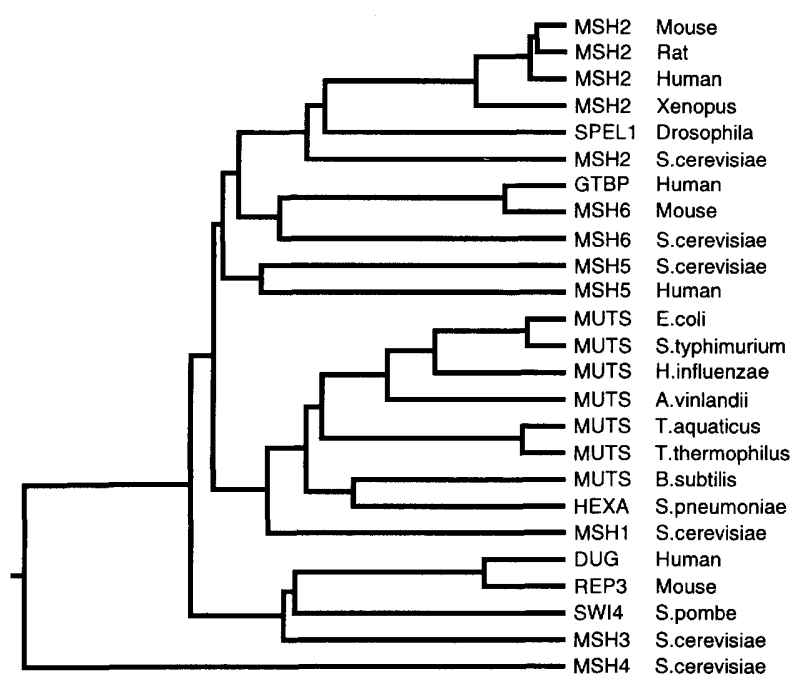

Figure 2. Family tree of known MutS homolog proteins. All of the sequences used for this construction were either retrieved from GenBank or are from unpublished studies; human MSH5, N. Winand and R. Kolodner (unpubl.); mouse MSH6, G. Crouse and R. Kolodner (unpubl.). 
Single-base mispair recognition

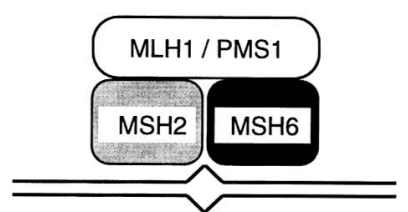

Insertion-deletion mispair recognition
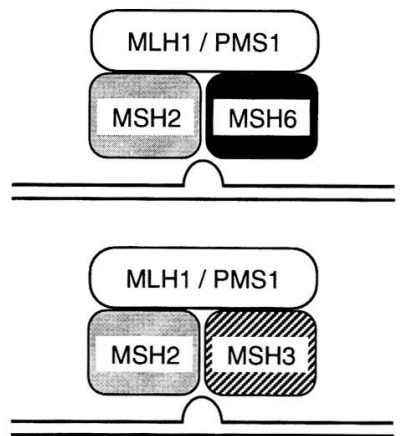

Figure 3. Model for mismatch recognition in $S$. cerevisiae. The various postulated complexes between $\mathrm{MSH} 2$ and either $\mathrm{MSH} 3$ or MSH6 are illustrated interacting with either a single-base substitution mispair or an insertion/deletion mispair; exactly which of the proteins in these complexes-MSH2, MSH3, or MSH6-actually interacts with the mispaired base is not known. Also indicated is the previously described MLH1-PMS1 complex that interacts with the mispair recognition complex. $T$ The $S$. cerevisiae protein names are given as primary names; the human protein names are the same except for PMSl, which is called PMS2 in humans, and MSH6, which has been called GTBP or pl60 in humans. (Reprinted, with permission, from Marsischky et al. 1996).

mutation assays that detect a broader variety of mutation events, and in these experiments, the mutation rate and mutation spectrum in the msh 3 msh 6 double mutant were essentially the same as that seen in the $m s h 2$ single mutant (Marsischky et al. 1996). These and other genetic data indicate that $\mathrm{MSH} 3$ and $\mathrm{MSH} 6$ encode redundant activities that act in $\mathrm{MSH2}$-dependent mismatch repair (Johnson et al. 1996; Marsischky et al. 1996). Consistent with this hypothesis, MSH2 forms heterodimeric complexes with both $\mathrm{MSH} 3$ and MSH6 proteins (Marsischky et al. 1996).

The analysis of $S$. cerevisiae MSH2, MSH3, and MSH6 has led to the proposal of a model in which there are two different pathways of $M S H 2$-dependent mismatch repair: repair that is primarily specific for single-base substitution mispairs and requires a MSH2-MSH6 complex, and repair that is primarily specific for insertion/deletion mispairs and requires either a MSH2-MSH3 complex or a MSH2-MSH6 complex (Fig. 3; Marsischky et al. 1996). The sum of the mutagenesis data reported suggests that repair of single-base substitution mispairs requires the MSH2-MSH6 complex, that repair of single-base insertion/deletion mispairs can utilize the MSH2-MSH6 or
MSH2-MSH3 complexes about equally, and that repair of larger insertion/deletion mispairs likely utilizes the MSH2-MSH3 complex 5-10 times as frequently as the MSH2-MSH6 complex (Johnson et al. 1996; Marsischky et al. 1996). A crucial test of this model will require the purification of these two complexes and the demonstration that they have the required mispair recognition properties.

The genetics of $S$. cerevisiae $\mathrm{MSH}$, $\mathrm{MSH}$, and $\mathrm{MSH} 6$, where comparable data exist, are consistent with the results of the analysis of human tumor cell lines. Like $S$. cerevisiae msh2 mutants, human msh2 mutant cell lines (e.g., LoVo, HEC59, 2774) have a strong general mutator phenotype and strong microsatellite instability including instability at dinucleotide and mononucleotide repeat loci (Bhattacharyya et al. 1994; Shibata etal. 1994; Boyer et al. 1995; Liu et al. 1995). Similar to the mutator phenotype of $S$. cerevisiae msh6 mutants, gtbp (msh6) mutant tumor lines (DLD1/HCT15, VACO543) have a strong mutator phenotype when the accumulation of mutations at HPRT (forward mutation assay) is measured, but their microsatellite instability phenotype is reduced compared to msh2 mutant tumor lines (Bhattacharyya et al. 1994; Shibata et al. 1994; Eshleman et al. 1995; Papadopoulos et al. 1995). Little is known about the possible presence of msh 3 mutations in either tumor cell lines or HNPCC families other than the fact that like GTBP (MSH6) mutations they must be rare because most HNPCC families studied have either msh2 or mlh1 mutations (Papadopoulos et al. 1995; Liu et al. 1996).

The genetic properties of MSH2, MSH3, and MSH6 in $S$. cerevisiae have important implications for the analysis of mismatch repair-defective mutations and their association with cancer susceptibility. First, the redundancy of $M S H 3$ and MSH6 compared to the apparently universal requirement for $M S H 2$ in mismatch repair provides an explanation for the high prevalence of msh2 mutations in HNPCC families compared to mutations in either GTBP (MSH6) or MSH3 (Papadopoulos et al. 1995; Liu et al. 1996). This is because independent mutations in both MSH3 and GTBP (MSH6) would be required to produce the same mismatch repair defect as that caused by mutations in MSH2. Second, selection for tumors and syndromes (HNPCC) associated with high degrees of microsatellite instability may preclude cancers and syndromes caused by msh 6 or $m s h 3$ mutations. In contrast, one might expect to find msh3 or $g t b p$ (msh6) mutations associated with cancers and cancer susceptibility syndromes with mutator phenotypes that more closely resemble the mutator phenotypes caused by $m s h 3$ and msh 6 mutations in S. cerevisiae. Third, it is possible that the mutator phenotype caused by msh 3 or gtbp (msh6) mutations compared to msh2 mutations is insufficient to cause cancer initiation or progression or that such mutations have lower penetrance than $m s h 2$ mutations. Fourth, it is possible that the lack of another entirely MSH2-dependent process, rather than the loss of mismatch repair, is the underlying cause of cancer susceptibility. Fifth, it is possible that there are tumors and tumor cell lines showing microsatellite instability that 
are $m s h 3, g t b p(m s h 6)$ double mutants. A particularly intriguing possibility is that mutations in $M S H 3$ and $G T B P(M S H 6)$ segregate in the human population and are relatively silent until $m s h 3 g t b p$ (msh6) double mutant individuals with increased cancer susceptibility are generated. It would require two independent events to inactivate the wild-type $M S H 3$ and GTBP (MSH6) alleles as expected in tumor cells from HNPCC individuals (Leach et al. 1993; Hemminki et al. 1994; Borresen et al. 1995) and the occurrence of two such events might be infrequent. However, the loss of the second allele of one of the genes, MSH3 or GTBP (MSH6), would cause a weak mutator phenotype that could speed inactivation of the remaining functional gene. It is also possible that $\mathrm{MSH} /$ msh3, GTBP (MSH6)/gtbp (msh6) cells might have an increased mutation rate resulting from unlinked partial noncomplementation, which would increase the loss of the second alleles of these genes.

\section{Homologs of the bacterial MutL proteins}

A variety of studies have documented a large number of eukaryotic genes encoding proteins that show a high degree of amino acid similarity with the bacterial MutL proteins (Figs. 3,4). It should be noted that the complete gene sequence is not known in all cases. Historically, these proteins have been designated as either PMS or MLH proteins because the first eukaryotic gene encoding a MutL homolog was named PMS1 before it had been cloned. These eukaryotic proteins, however, are all related to the bacterial MutL proteins. The available evidence suggests that at least two of these proteins, MLH1 and PMS1 (PMS2 in humans), are involved in mismatch repair. The involvement of two such proteins in mismatch repair, as compared to the involvement of a single MutL protein in bacteria, parallels the complexity of eu-

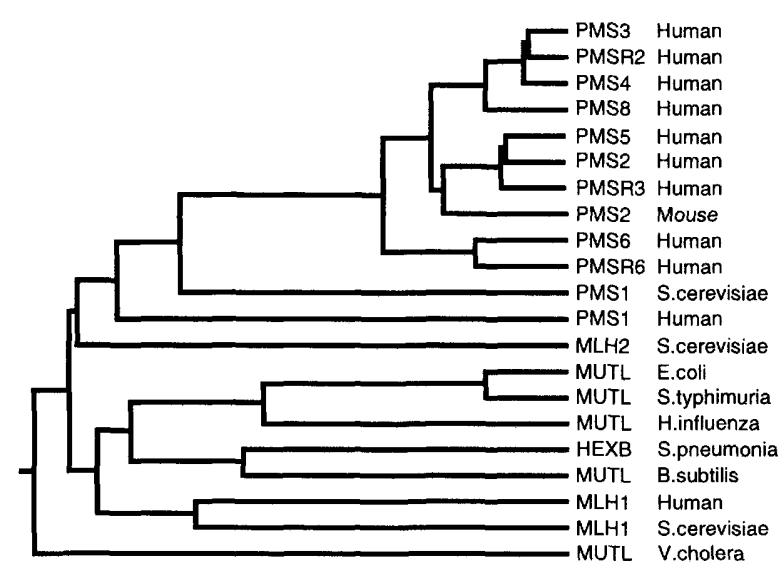

Figure 4. Family tree of known MutL homolog proteins. All of the sequences used for this construction were retrieved from GenBank except $S$. cerevisiae $M L H 2$, which was provided by Tom Prolla and R. Michael Liskay (Prolla 1994). Some of the sequences used (hPMS5, hPMS6, hPMSR6, hPMS8) are not complete coding sequences. karyotic mismatch repair seen in regard to the MSH proteins.

In both $S$. cerevisiae and human cells, there appear to be at least two MutL homologs that are required for mismatch repair: MLH1 and PMS1 in S. cerevisiae, and MLH1 and PMS2 (the closest homolog of $S$. cerevisiae PMS1) in human cells (Prolla et al. 1994; Kolodner 1995; Li and Modrich 1995; Modrich and Lahue 1996). These proteins appear to exist in a 1:1 complex (Prolla et al. 1994b; Li and Modrich 1995). The purified human MLH1-PMS2 complex complements the in vitro mismatch repair defect caused by mlh1 mutations found in human tumor cell lines, indicating that it is functional in mismatch repair ( $\mathrm{Li}$ and Modrich 1995). In S. cerevisiae, this MLH1-PMS1 complex interacts with MSH2 bound to a mispaired base, consistent with the idea that the MLH1-PMS1 (human PMS2) complex plays the same role in mismatch repair in eukaryotes that MutL plays in bacteria. The available biochemical and genetic data (discussed in more detail below) and the various protein sequence homology relationships support the idea that the $S$. cerevisiae MLH1-PMS1 and human MLH1-PMS2 complexes play equivalent roles in mismatch repair.

In human cells there is a third MutL homolog, PMS1, that has been implicated in mismatch repair by virtue of the observation of a germ-line mutation in PMS1 in a single patient with a family history of colon cancer (Nicolaides et al. 1994). The tumor from this patient was not examined for either loss of the second PMS1 allele or for microsatellite instability. Therefore, it is not clear whether PMS1 behaves like other mismatch repair and tumor suppressor genes during tumor development or whether pms 1 mutations cause a mismatch repair defect in tumors. At present, little is known about the biochemical role of hPMS1 in mismatch repair. Additional studies of human PMS1 are clearly needed.

The genetics of S. cerevisiae PMS1 and MLH1 and human $M L H 1$ and PMS2 parallel each other to a great extent. The available data indicate that mutations in $S$. cerevisiae MLH1 and PMS1 cause a similar, strong general mutator phenotype that is virtually the same as that caused by mutations in $\mathrm{MSH} 2$; no differences in the mutator phenotypes caused by pms 1 and $m l h 1$ mutations have yet been published (Williamson et al. 1985; Bishop et al. 1987; B. Kramer et al. 1989; Strand et al. 1993; Prolla et al. 1994a; Jeyaprakash et al. 1996). Interestingly, in a recent study on the effects of $m s h 2, m s h 3$, and $p m s 1$ (human pms2) mutations on crossing over between divergent DNA sequences, PMS1 (human PMS2) appeared to play significantly less of a role in suppressing homologous recombination than MSH2 (Datta et al. 1996; Hunter et al. 1996). This suggests that defects in PMS1 (human PMS2) might not be equivalent to defects in other mismatch repair genes (unfortunately $M L H 1$ was not evaluated in these studies; Datta et al. 1996; Hunter et al. 1996). Analysis of human tumor cell lines with mutations in either MLH1 or PMS2 support this view: Such cell lines have a strong, global microsatellite instability phenotype, and extracts prepared from such cell 
lines are mismatch repair defective (Parsons et al. 1993; Bhattacharyya et al. 1994; Shibata et al. 1994; Boyer et al. 1995; Li and Modrich 1995; Risinger et al. 1995). Furthermore, the mismatch repair defect caused by mlh1 mutations can be complemented in vitro by the addition of the MLH1-PMS2 complex (Li and Modrich 1995). In addition, cells derived from homozygous pms2 mutant mice show microsatellite instability, consistent with a mismatch repair defect (Baker et al. 1995). One striking feature about the genetics of the human $M L H 1$ and PMS2 genes is that there is a strikingly lower frequency of germ-line pms 2 mutations compared to germ-line mlh1 mutations in HNPCC families, especially given that mutations in both genes appear to cause the same type of mismatch repair defect (Boyer et al. 1995; Risinger et al. 1995; Liu et al. 1996). These data may reflect trivial possibilities such as founder effects (NystromLahti et al. 1995), or DNA sequence contexts may make MLH1 more mutable than PMS2. Alternately, these data raise the intriguing possibilities that $M L H 1$ and $P M S 2$ do not play equivalent roles in the cell even though they are subunits of the same complex (see above discussion of Datta et al. 1996; Hunter et al. 1996), that pms2 mutations confer a selective disadvantage to the population compared to mlh1 mutations (Baker et al. 1995), or that like S. cerevisiae MSH3 and MSH6, there is a function that is redundant with PMS2. The resolution of this question could provide important information about how mismatch repair defects give rise to cancer susceptibility.

\section{Other components required for mismatch repair}

It seems clear that HNPCC families, sporadic tumors, and tumor cell lines that are associated with mismatch repair defects or that exhibit microsatellite instability exist where it has not yet been possible to demonstrate defects in known mismatch repair genes (da Costa et al. 1995; Katabuchi et al. 1995; Liu et al. 1995, 1996; Papadopoulos et al. 1995; Wijnen et al. 1995, 1996). This observation has provided additional interest in the identification of other components of eukaryotic mismatch repair. At present, there is little definitive additional information about the other proteins that function in eukaryotic MutHLS-like mismatch repair pathways. On the basis of our current understanding of the enzymes that promote MutHLS mismatch repair in E. coli, obvious candidates for proteins required for eukaryotic mismatch repair are exonucleases, helicases, and enzymes required for DNA synthesis (Modrich 1991; Kolodner 1995; Modrich and Lahue 1996). A particularly interesting unresolved issue is the identification of the proteins and signals that function in the recognition of the newly replicated DNA strand during mismatch repair; this is because DNA methylation and MutH homologs seem unlikely to play this role as they do in E. coli (Modrich 1991; Kolodner 1995; Modrich and Lahue 1996). Whereas most of the progress reported in eukaryotic mismatch repair enzymology has been in the area of the MSH and MLH proteins, there has been some recent progress in the identification of exonucleases that might play a role in eukaryotic mismatch repair.

A $5^{\prime} \rightarrow 3^{\prime}$ endo/exonuclease, $S$. cerevisiae RTH1 (RAD27, YKL510), has been proposed to function in mismatch repair (Johnson et al. 1995). Mutations in RTH1 (RAD27) cause a mutator phenotype (Johnson et al. 1995; Reagan et al. 1995; Vallen and Cross 1995) that in one study was shown to be as strong as that caused by $\mathrm{mu}$ tations in MSH2, MLH1, and PMS1 (Johnson et al. 1995). This result seems at odds with mechanistic studies of mismatch repair promoted by human cell extracts indicating that, like the E. coli reaction, eukaryotic mismatch repair is likely to involve redundant $5^{\prime} \rightarrow 3^{\prime}$ and $3^{\prime} \rightarrow 5^{\prime}$ exonucleases (Modrich 1991; Fang and Modrich 1993; Modrich and Lahue 1996). Double mutant combinations of $r$ th1 and either msh2, mlh1, or pms1 showed a three- to fivefold synergistic effect (Johnson et al. 1995). One possible interpretation of this result is that RTH1 functions in an entirely different pathway from $M S H 2$, $M L H 1$, and PMS1. Additional genetic analysis of $R T H 1$ (RAD27) has indicated that $R T H 1$ (RAD27) plays a role in repair of other types of DNA damage, that $R T H 1$ (RAD27) is a member of the RAD6 epistasis group that functions in DNA damage tolerance, and that rth1 (rad27) mutants also have a cell cycle defect (Reagan et al. 1995; Vallen and Cross 1995). Studies of the S. cerevisiae (called RTH1, RAD27, YKL510) and human (called FEN-1, MF-1, exonuclease IV) protein have suggested that RTH1 functions in the processing of $5^{\prime}$ ends of Okazaki fragments and processing branched DNA structures formed by different DNA repair pathways (Ishimi et al. 1988; Harrington and Lieber 1994; Waga and Stillman 1994; Waga et al. 1994; Hiraoka et al. 1995; Sommers et al. 1995). There are several possible interpretations of the data on RTH1 with regard to a possible role in mismatch repair. (1) RTH1 functions as an exonuclease during mismatch repair; however, the strong mutator phenotype of $r$ th1 mutants seems inconsistent with the mechanistic studies implicating bidirectional excision during mismatch repair. This suggests that if RTHl functions in mismatch repair, it likely functions in other repair reactions that act to reduce mutation rates. (2) RTH1 might not function as an exonuclease but rather as an endonuclease that cleaves branched structures that must be processed during different DNA repair pathways, possibly including mismatch repair. (3) The mutator phenotype seen in rth1 mutants is not attributable to a mismatch repair defect but is rather the result of defects in the fidelity of DNA synthesis, or other repair defects. This latter possibility could account for the modest synergistic effect of rth1 mutations in combination with $m s h 2$, mlh1, and pms1 mutations. Clearly caution must be taken in the interpretation of the meaning of mutator phenotypes.

A second candidate for an exonuclease involved in mismatch repair was identified in a two-hybrid screen with $S$. cerevisiae MSH2 as a bait protein (D.X. Tishkoff, A. Beddow, M.F. Kane, and R. Kolodner, in prep.). The $S$. cerevisiae EXO1 gene was identified as encoding a protein that interacts with both $S$. cerevisiae and human 
MSH2, and a $S$. cerevisiae MSH2-EXO1 complex was identified in vivo by coimmunoprecipitation. $S$. cerevisiae EXOl appears to be a homolog of the Schizosaccharomyces pombe $5^{\prime} \rightarrow 3^{\prime}$ exonuclease EXOl (Szankasi and Smith 1992, 1995; D.X. Tishkoff, A. Beddow, M.F. Kane, and R. Kolodner, in prep.), and characterization of the overproduced and purified $S$. cerevisiae EXO1 has indicated that it preferentially degrades double-stranded DNA compared with single-stranded DNA. Mutations in $S$. cerevisiae EXO1 cause a weak general mutator phenotype and low levels of microsatellite instability, consistent with the redundant exonuclease proposal. Furthermore, analysis of double mutants was consistent with EXO1 functioning in a MSH2-dependent pathway (D.X. Tishkoff, A. Beddow, M.F. Kane, and R. Kolodner, in prep.). S. pombe exo1 mutants also have a mutator phenotype, and $S$. pombe EXOl has been proposed to function in either a general MutHLS-like mismatch repair system or a more specific mismatch repair system like the base-specific systems described in bacteria (Szankasi and Smith 1995). At this stage of analysis additional studies are clearly needed to define the potential roles of RTH1 (RAD27) and EXOl in mismatch repair and to identify additional exonucleases that might be required.

\section{Is there another eukaryotic general mismatch repair pathway?}

A number of lines of investigation have suggested that there may be another eukaryotic mismatch repair pathway that has an overlapping specificity with the MutHLS-like pathway. When gene conversion was analyzed in $S$. cerevisiae pms1, msh2, or mlh1 mutants by use of tetrad analysis, it was observed that there was a low, residual level of gene conversion independent of the marker analyzed /single-base substitution mutations or small insertion/deletion mutations) (Williamson et al. 1985; Reenan and Kolodner 1992a; Alani et al. 1994; Prolla et al. 1994a). This could occur for two possible reasons: Either a proportion of gene conversion events are caused by gap repair and do not involve the formation of a heteroduplex recombination intermediate (discussed in Alani et al. 1994), or there is an inefficient mismatch repair pathway functional in pms1, msh2, or mlh1 null mutants. Similarly, when transformation assays were used to analyze mismatch repair directly, not all apparent mismatch repair was eliminated by mutations in mismatch repair genes (Bishop et al. 1987, 1989; B. Kramer et al. 1989|. Furthermore, the frequency of insertion events approximately equaled the frequency of deletion events when insertion/deletion mispairs were repaired after transformation of wild-type $S$. cerevisiae cells, whereas there was a strong disparity in favor of deletion events among the fraction of insertion/deletion mispairs that appeared to be repaired in a pms1 mutant (Bishop and Kolodner 1986; Bishop et al. 1987). A similar shift toward deletion events in mismatch repair mutants was seen when alterations in GT tract length resulting from replication errors were analyzed (Strand et al. 1993,
1995). One of several possible explanations for these data is that there could be a separate, less-efficient repair pathway that selectively repairs insertion/deletion mispairs by deletion. Consistent with this view, analysis of mismatch repair catalyzed in extracts of human tumor cell lines has suggested there may be an $M L H 1$-independent mismatch repair pathway that promotes the repair of some insertion/deletion mispairs $>3$ nucleotides in length and that repair of some such substrates may also be MSH2-independent (Umar et al. 1994a).

More recently, several workers have detected $S$. cerevisiae and human mispair binding activities present in $S$. cerevisiae msh2, $m s h 3$, and $m s h 4$ mutants and human msh2 and gtbp (msh6) mutants (Miret et al. 1996; $\mathrm{O}^{\prime}$ Regan et al. 1996). Other studies have detected activities that nick DNA at the site of a mispair (Chang and Lu 1991; Yao and Kom 1994; Yeh et al. 1994). However, it has not been established whether these activities play a role in a general mismatch repair pathway such as the MutHLS-like pathway or whether these activities act in other pathways such as crossing over during meiotic recombination, which is known to require MSH4 and MSH5 (Ross-Macdonald and Roeder 1994; Hollingsworth et al. 1995), or in base-specific mismatch repair (Modrich 1991; Kolodner 1995; Modrich and Lahue 1996). Whether these types of activities play roles in other mismatch repair pathways and whether alternate mismatch repair pathways that recognize a broad spectrum of mispaired bases including larger insertion/deletion mispairs exist remain important unanswered questions at this time.

\section{Conclusions}

A considerable amount of progress has been made in the identification of components of eukaryotic MutHLS-like mismatch repair pathways. These pathways, however, appear more complex than their bacterial counterparts. Furthermore, not all of the proteins that function in these eukaryotic pathways have been identified, nor has it been resolved how many such repair pathways might exist. Particular challenges for the future include the complete identification of the components of these repair pathways, the understanding of how defects in these pathways lead to cancer susceptibility, and the unraveling of the genetics of these pathways in model organisms, which may provide greater insights into the role of inherited and acquired repair defects in cancer susceptibility in the human population.

\section{Acknowledgments}

I thank Richard Boland, Tom Kunkel, Gerry Marsischky, Dan Tishkoff, Geoff Wahl, and Jean Wang for suggestions and comments on the manuscript. I particularly acknowledge the hospitality of the Salk Institute for Biological Studies during the preparation of this paper. Work in my laboratory on mismatch repair has been supported by National Institutes of Health grants GM50006 and CA67151. 


\section{References}

Alani, E., R.A.G. Reenan, and R. Kolodner. 1994. Mismatch repair proteins directly affect gene conversion in Saccharomyces cerevisiae by regulating heteroduplex tract length. Genetics 137: 19-39.

Alani, E., N.-W. Chi, and R.D. Kolodner. 1995. The Saccharomyces cerevisiae MSH2 protein specifically binds to duplex oligonucleotides containing mismatched DNA base pairs and loop insertions. Genes \& Dev. 9: 234-247.

Anthoney, D.A., A.J. McIlwrath, W.M. Gallagher, A.R.M. Edlin, and R. Brown. 1996. Microsatellite instability, apoptosis, and loss of p53 function in drug-resistant tumor cells. Cancer Res. 56: 1374-1381.

Baker, S.M., C.E. Bronner, I. Zhang, A. Plug, M. Robatzek, G. Warren, E.A. Elliott, J. Yu, T. Ashley, N. Arnheim, R.A. Flavell, and R.M. Liskay. 1995. Male mice defective in the DNA mismatch repair gene PMS2 exhibit abnormal chromosome synapsis in meiosis. Cell 82: 309-320.

Bhattacharyya, N.P., A. Skandalis, A. Ganesh, J. Groden, and M. Meuth. 1994. Mutator phenotype in human colorectal carcinoma cell lines. Proc. Natl. Acad. Sci. 91: 6319-6323.

Bishop, D.K. and R.D. Kolodner. 1986. Repair of heteroduplex plasmid DNA after transformation into Saccharomyces cerevisiae. Mol. Cell. Biol. 6: 3401-3409.

Bishop, D.K., M.S. Williamson, S. Fogel, and R.D. Kolodner. 1987. The role of heteroduplex correction in gene conversion in Saccharomyces cerevisiae. Nature 328: 362-364.

Bishop, D.K., J. Andersen, and R.D. Kolodner. 1989. Specificity of mismatch repair following transformation of Saccharomyces cerevisiae with heteroduplex plasmid DNA. Proc. Natl. Acad. Sci. 86: 3713-3717.

Borresen, A.-L., R.A. Lothe, G.I. Meling, S. Lystad, P. Morrison, J. Lipford, M.F. Kane, T.O. Rognum, and R.D. Kolodner. 1995. Somatic mutations in the hMSH2 gene in microsatellite unstable colorectal carcinomas. Hum. Mol. Genet. 11: 2065-2072.

Boyer, J.C., A. Umar, J. Risinger, M.F. Kane, J. Lipford, J.C. Barrett, R.D. Kolodner, and T.A. Kunkel. 1995. Microsatellite instability, mismatch repair deficiency and genetic defects in human cancer cell lines. Cancer Res. 55: 6062-6070.

Bronner, C.E., S.M. Baker, P.T. Morrison, G. Warren, L.G. Smith, M.K. Lescoe, M. Kane, C. Earabino, J. Lipford, A. Lindblom, P. Tannergard, R.J. Bollag, A.R. Godwin, D.C. Ward, M. Nordenskjold, R. Fishel, R. Kolodner, and R.M. Liskay. 1994. Mutation in the DNA mismatch repair gene homologue $h M L H 1$ is associated with hereditary nonpolyposis colon cancer. Nature 368: 258-261.

Chang, D.Y. and A.-L. Lu. 1991. Base mismatch-specific endonuclease activity in extracts from Saccharomyces cerevisiae. Nucleic Acids Res. 19: 4761-4766.

da Costa, L.T., B. Liu, W. el-Deiry, S.R. Hamilton, K.W. Kinzler, B. Vogelstein, S. Markowitz, J.K. Willson, A. de la Chapelle, K.M. Downey, and A.G. So. 1995. Polymerase delta variants in RER colorectal tumours [letter]. Nature Genet. 9: 10-11.

Datta, A., A. Adjiri, L. New, G.F. Crouse, and S. Jinks-Robertson. 1996. Crossovers between diverged sequences are regulated by mismatch repair proteins in yeast. Mol. Cell. Biol. 16: 1085-1093.

Drummond, J.T., G.-M. Li, M.J. Longley, and P. Modrich. 1995. Mismatch recognition by an hMSH2-GTBP heterodimer and differential repair defects in tumor cells. Science 268: 1909-1912.

Eshleman, J.R. and S.D. Markowitz. 1995. Microsatellite instability in inherited and sporadic neoplasms. Cur. Opin. Oncol. 7: 83-89.
Eshleman, J.R., E.Z. Lang, G.K. Bowerfind, R. Parsons, B. Vogelstein, J.K.V. Wilson, M.L. Veigl, W.D. Sedwick, and S.D. Markowitz. 1995. Increased mutation rate at the hprt locus accompanies microsatellite instability in colon cancer. Oncogene 10: 33-37.

Fang, W.-H. and P. Modrich. 1993. Human strand-specific mismatch repair occurs by a bidirectional mechanism similar to that of the bacterial reaction. J. Biol. Chem. 268: 11838-11844.

Feng, W.Y., E.H. Lee, and J.B. Hays. 1991. Recombinogenic processing of UV-light photoproducts in nonreplicating phage DNA by the Escherichia coli methyl-directed mismatch repair system. Genetics 129: 1007-1020.

Fishel, R. and R.D. Kolodner. 1995. Identification of mismatch repair genes and their role in the development of cancer. Curr. Opin. Genet. Dev. 5: 382-395.

Fishel, R.A., M.K. Lescoe, M.R.S. Rao, N. Copland, N. Jenkins, J. Garber, M. Kane, and R. Kolodner. 1993. The human mutator gene homolog MSH2 and its association with hereditary nonpolyposis colon cancer. Cell 75: 1027-1038.

Fishel, R.A., A. Ewel, S. Lee, M.K. Lescoe, and J. Griffith. 1994. Binding of mismatched microsatellite DNA sequences by the human MSH2 protein. Science 266: 1403-1405.

Fram, R.J., P.S. Cusick, J.M. Wilson, and M.G. Marinus. 1985. Mismatch repair of cis-Diamminedichloroplatinum(II)-Induced DNA damage. Mol. Pharmacol. 28: 51-55.

Friedberg, E.C., G.C. Walker, and W. Siede. 1995. DNA repair and mutagenesis. ASM Press, Washington, D.C.

Grilley, M., J. Griffith, and P. Modrich. 1993. Bidirectional excision in methyl-directed mismatch repair. J. Biol. Chem. 268: 11830-11837.

Harrington, J.J. and M.R. Lieber. 1994. Functional domains within FEN-1 and RAD2 define a family of structure-specific endonucleases: Implications for nucleotide excision repair. Genes \& Dev. 8: 1344-1355.

Hawn, M.T., A. Umar, J.M. Caretthers, G. Marra, T.A. Kunkel, R.C. Boland, and M. Koi. 1995. Evidence for a connection between the mismatch repair system and the $\mathrm{G} 2$ cell cycle checkpoint. Cancer Res. 55: 3721-3725.

Hemminki, A., P. Peltomaki, J.-P. Mecklin, H. Jarvinen, R. Salovaara, M. Nystrom-Lahti, A. de la Chapelle, and L.A. Aaltonen. 1994. Loss of the wild-type MLH1 gene is a feature of hereditary nonpolyposis colorectal cancer. Nature Genet. 8: $405-411$.

Hiraoka, L.R., J.J. Harrington, D.S. Gerhard, M.R. Lieber, and C.-L. Hsieh. 1995. Sequence of human FEN-1, a structurespecific endonuclease, and chromosomal location of the gene (FEN1) in mouse and human. Genomics 25: 220-225.

Hollingsworth, N.M., L. Ponte, and C. Halsey. 1995. MSH5, a novel MutS homolog, facilitates meiotic reciprocal recombination between homologs in Saccharomyces cerevisiae but not mismatch repair. Genes \& Dev. 9: 1728-1739.

Hunter, N., S.R. Chambers, E.J. Louis, and R.H. Borts. 1996. The mismatch repair system contributes to meiotic sterility in an interspecific yeast hybrid. $E M B O /$. 15: 1726-1733.

Ishimi, Y., A. Claude, P. Bullock, and J. Hurwitz. 1988. Complete enzymatic synthesis of DNA containing the SV40 origin of replication. J. Biol. Chem. 263: 19723-19733.

Jeyaprakash, A., R. DasGupta, and R. Kolodner. 1996. Saccharomyces cerevisiae pms 2 mutations are alleles of $M L H 1$ and pms2-2 corresponds to an HNPCC causing missense mutation. Mol. Cell Biol. (in press).

Johnson, R.E., K.K. Gopala, L. Prakash, and S. Prakash. 1995. Requirement of the yeast RTH1 $5^{\prime}$ to $3^{\prime}$ exonuclease for the stability of simple repetitive DNA. Science 269: 238-240.

Johnson, R.E., G.K. Kovvali, L. Prakash, and S. Prakash. 1996. Requirement of the yeast $\mathrm{MSH} 3$ and $\mathrm{MSH} 6$ genes for MSH2- 
dependent genomic stability. J. Biol. Chem. 271: 7285-7288. Karran, P. and M.G. Marinus. 1982. Mismatch correction of $\mathrm{O}^{6}$-methylguanine residues in $E$. coli DNA. Nature 296: 868-869.

Katabuchi, H., B. van Rees, A.R. Lambers, B.M. Ronnett, M.S. Blazes, F.S. Leach, K.R. Cho, and L. Hedrick. 1995. Mutations in DNA mismatch repair genes are not responsible for microsatellite instability in most sporadic endometrial carcinomas. Cancer Res. 55: 5556-5560.

Kolodner, R.D. 1995. Mismatch repair: Mechanisms and relationship to cancer susceptibility. Trends Biochem. 20:397-402.

Kramer, B., W. Kramer, M.S. Williamson, and S. Fogel. 1989. Heteroduplex DNA correction in Saccharomyces cerevisiae is mismatch specific and requires functional PMS genes. Mol. Cell. Biol. 9: 4432-4440.

Kramer, W., B. Kramer, M.S. Williamson, and S. Fogel. 1989. Cloning and nucleotide sequence of DNA mismatch repair gene PMS1 from Saccharomyces cerevisiae: Homology of PMS1 to procaryotic MutL and HexB. J. Bacteriol. 171: 5339-5346.

Lahue, R.S., K.G. Au, and P. Modrich. 1989. DNA mismatch correction in a defined system. Science 245: 160-164.

Leach, F.S., N.C. Nicolaides, N. Papadopoulos, B. Liu, J. Jen, R. Parsons, P. Peltomaki, P. Sistonen, L.A. Aaltonen, M. Nystrom-Lahti, X.-Y. Guan, J. Zhang, P.S Meltzer, J.-W. Yu, F.-T. Kao, D.J. Chen, K.M. Cerosaletti, R.E.K. Fournier, S. Todd, T. Lewis, R.J. Leach, S.L. Naylor, J. Weissenbach, J.-P. Mecklin, H. Jarvinen, G.M. Petersen, S.R. Hamilton, J. Green, J. Jass, P. Watson, H.T. Lynch, J.M. Trent, A. de la Chapelle, K.W. Kinsler, and B. Vogelstein. 1993. Mutations of a mutS homolog in hereditary nonpolyposis colorectal cancer. Cell 75: 1215-1225.

Li, G.-M. and P. Modrich. 1995. Restoration of mismatch repair to nuclear extracts of $\mathrm{H} 6$ colorectal tumor cells by a heterodimer of human MutL homologs. Proc. Natl. Acad. Sci. 92: 1950-1954.

Liu, B., M.C. Nicolaides, S. Markowitz, J.K.V. Willson, J.R. Parsons, J. Jen, N. Papadopolous, P. Peltomaki, A. de la Chapelle, S.R. Hamilton, K.W. Kinzler, and B. Vogelstein. 1995. Mismatch repair gene defects in sporadic colorectal cancers with microsatellite instability. Nature Genet. 9: 4855.

Liu, B., R. Parsons, N. Papadopoulos, N.C. Nicolaides, H.T. Lynch, P. Watson, J.R. Jass, M. Dunlop, A. Wyllie, P. Peltomaki, A. de la Chapelle, S.R. Hamilton, R. Vogelstein, and K.W. Kinzler. 1996. Analysis of mismatch repair genes in hereditary non-polyposis colorectal cancer patients. Nature Med. 2: 169-174.

Marra, G. and C.R. Boland. 1995. Hereditary nonpolyposis colorectal cancer: The syndrome, the genes and historical perspectives. J. Natl. Cancer Inst. 87: 1114-1125.

Marsischky, G.T., N. Filosi, M.F. Kane, and R. Kolodner. 1996. Redundancy of Saccharomyces cerevisiae MSH3 and MSH6 in MSH2-dependent mismatch repair. Genes \& Dev. 10: $407-420$.

Mellon, I. and G.N. Champe. 1996. Products of DNA mismatch repair genes mutS and mutL are required for transcriptioncoupled nucleotide excision repair of the lactose operon in Escherichia coli. Proc. Natl. Acad. Sci. 93: 1292-1297.

Miret, J.J., B.O. Parker, and R.S. Lahue. 1996. Recognition of DNA insertion/deletion mismatches by an activity in Saccharomyces cerevisiae. Nucleic Acids Res. 24: 721-729.

Modrich, P. 1991. Mechanisms and biological effects of mismatch repair. Annu. Rev. Genet. 25: 229-253.

Modrich, P. and R. Lahue. 1996. Mismatch repair in replication fidelity, genetic recombination and cancer biology. Annu.
Rev. Biochem. 65: 101-133.

New, L., K. Liu, and G.F. Crouse. 1993. The yeast gene MSH3 defines a new class of eukaryotic MutS homologues. Mol. \& Gen. Genet. 239: 97-108.

Nicolaides, N.C., N. Papadopoulos, B. Liu, Y. Wei, K.C. Carter, S.M. Ruben, C.A. Rosen, W.A. Haseltine, R.D. Fleischmann, C.M. Fraser, M.D. Adams, J.C. Venter, M.G. Dunlop, S.R. Hamilton, G.M. Petersen, A. de la Chapelle, B. Vogelstein, and K. Kinzler. 1994. Mutations of two PMS homologues in hereditary nonpolyposis colon cancer. Nature 371: 75-80.

Nystrom-Lahti, M., P. Kristo, N.C. Nicoladies, S.-Y. Chang, L.A. Aaltonen, A.-L. Moisio, H.J. Jarvinen, J.-P. Mecklin, K. Kinzler, B. Vogelstein, A. de la Chapelle, and P. Peltomaki. 1995. Founding mutations and Alu-mediated recombination in hereditary colon cancer. Nature Med. 1: 6054-6058.

O'Regan, N.E., P. Branch, P. Macpherson, and P. Karran. 1996. hMSH2-independent DNA mismatch recognition by human proteins. J. Biol. Chem. 271: 1789-1796.

Palombo, F., P. Gallinari, I. Iaccarino, T. Lettieri, M. Hughes, A. D'Arrigo, O. Truong, J.J. Hsuan, and J. Jiricny. 1995. GTBP, a $160 \mathrm{kD}$ protein essential for mismatch binding activity in human cells. Science 268: 1912-1914.

Papadopoulos, N., N.C. Nicolaides, Y.-F. Wei, S.M. Ruben, K.C. Carter, C.A. Rosen, W.A. Haseltine, R.D. Fleischmann, C.M. Fraser, M.D. Adams, J.C. Venter, S.R. Hamilton, G.M. Petersen, P. Watson, H.T. Lynch, P. Peltomaki, J.-P. Mecklin, A. de la Chapelle, K.W. Kinzler, and B. Vogelstein. 1994. Mutation of a mutL homolog in hereditary colon cancer. Science 263: 1625-1629.

Papadopoulos, N., N.C. Nicolaides, B. Liu, R.E. Parsons, C. Lengauer, F. Palombo, A. D'Arrigo, S. Markowitz, J.K.V. Wilson, K.W. Kinzler, J. Jiricny, and B. Vogelstein. 1995. Mutations of GTBP in genetically unstable tumors. Science 268: 19151917.

Parsons, R., G.-M. Li, M.J. Longley, W.-H. Fang, N. Papadopoulos, J. Jen, A. de la Chapelle, K.W. Kinzler, B. Vogelstein, and P. Modrich. 1993. Hypermutability and mismatch repair deficiency in RER + tumor cells. Cell 75: 1227-1236.

Proffitt, J.H., J.R. Davie, D. Swinton, and S. Hattman. 1984. 5-Methylcytosine is not detectable in Saccharomyces cerevisiae. Mol. Cell. Biol. 4: 985-988.

Prolla, T.A. 1994. Dual requirement in yeast DNA mismatch repair for MLH1 and PMS1, two homologs of bacterial MutL. Ph.D. thesis. Yale University, New Haven, CT.

Prolla, T.A., D.-M. Christie, and R.M. Liskay. 1994a. A requirement in yeast DNA mismatch repair for MLH1 and PMS1, two homologs of the bacterial mutL gene. Mol. Cell. Biol. 14: 407-415.

Prolla, T.A., Q. Pang, E. Alani, R.D. Kolodner, and R.M. Liskay. 1994b. Interactions between the MSH2, MLH1 and PMS1 proteins during the initiation of DNA mismatch repair. Science 265: 1091-1093.

Rayssiguier, C., D.S. Thaler, and M. Radman. 1989. The barrier to recombination between Escherichia coli and Salmonella typhimurium is disrupted in mismatch-repair mutants. $\mathrm{Na}$ ture 342: 396-401.

Reagan, M.S., C. Pittenger, W. Siede, and E.C. Friedberg. 1995. Characterization of a mutant strain of Saccharomyces cerevisiae with a deletion of the RAD27 gene, a structural homologog of the RAD2 nucleotide excision repair gene. J. Bacteriol. 177: 364-371.

Reenan, R.A.G. and R.D. Kolodner. 1992a. Characterization of insertion mutations in the Saccharomyces cerevisiae MSH1 and $\mathrm{MSH} 2$ genes: Evidence for separate mitochondrial and nuclear functions. Genetics 132: 975-985.

- 1992b. Isolation and characterization of two Saccharo- 
myces cerevisiae genes encoding homologs of the bacterial HexA and MutS mismatch repair proteins. Genetics 132: 963-973.

Risinger, J.I., A. Umar, J.C. Barrett, and T.A. Kunkel. 1995. A hPMS2 mutant cell line is defective in strand-specific mismatch repair. J. Biol. Chem. 270: 18183-18186.

Ross-Macdonald, P. and G.S. Roeder. 1994. Mutation of a meiosis-specific MutS homolog decreases crossing over but not mismatch correction. Cell 79: 1069-1080.

Selva, E.M., L. New, G.F. Crouse, and R.S. Lahue. 1995. Mismatch correction acts as a barrier to homeologous recombination in Saccharomyces cerevisiae. Genetics 139: 11751188.

Shibata, D., M.A. Peinado, S. Ionov, S. Malkhosyan, and M. Perucho. 1994. Genomic instability in repeated sequences in an early somatic event in colorectal tumorigenesis that persists after transformation. Nature Genet. 6: 273-281.

Sommers, C.H., E.J. Miller, B. Dujon, S. Prakash, and L. Prakash. 1995. Conditional lethality of null mutations in RTH1 that encodes the yeast counterpart of a mammalian 5'-to 3 '-exonuclease required for lagging strand DNA synthesis in reconstituted systems. I. Biol. Chem. 270: 4193-4196.

Strand, M., T.A. Prolla, R.M. Liskay, and T.D. Petes. 1993. Destabilization of tracts of simple repetitive DNA in yeast by mutations affecting DNA mismatch repair. Nature 365: 274-276.

Strand, M., M.C. Earley, G.F. Crouse, and T.D. Petes. 1995. Mutations in the $\mathrm{MSH} 3$ gene preferentially lead to deletions within tracts of simple repetitive DNA in Saccharomyces cerevisiae. Proc. Natl. Acad. Sci. 92: 10418-10421.

Szankasi, P. and G.R. Smith. 1992. A DNA exonuclease induced during meiosis of Schizosaccharomyces pombe. I. Biol. Chem. 267: 3014-3023.

-1995. A role for exonuclease I from $S$. pombe in mutation avoidance and mismatch correction. Science 267: 11661169.

Umar, A., J.C. Boyer, and T.A. Kunkel. 1994a. DNA loop repair by human cell extracts. Science 266: 814-816.

Umar, A., J.C. Boyer, D.C. Thomas, D.C. Nguyen, J.I. Risinger, J. Boyd, Y. Ionov, M. Perucho, and T.A. Kunkel. 1994b. Defective mismatch repair in extracts of colorectal and endometrial cancer cell lines exhibiting microsatellite instability. $I$. Biol. Chem. 269: 14367-14370.

Vallen, E.A. and R.R. Cross. 1995. Mutations in RAD27 define a potential link between G1 cyclins and DNA replication. Mol. Cell Biol. 15: 4291-4302.

Waga, S. and B. Stillman. 1994. Anatomy of a DNA replication fork revealed by reconstitution of SV40 DNA replication in vitro. Nature 369: 207-212.

Waga, S., G. Bauer, and B. Stillman. 1994. Reconstitution of complete SV40 replication with purified replication factors. I. Biol. Chem. 269: 10923-10934.

Watanabe, A., M. Ikejima, N. Suzuki, and T. Shimada. 1996. Genomic organization and expression of the human $\mathrm{MSH} 3$ gene. Genomics 31: 311-318.

Wijnen, J., H. Vassen, P. Meera Kahn, F.H. Menko, H. van der Klift, C. van Leeuwen, M. van den Broek, I. van LeeuwenCornelisse, F. Nagengast, A. Meijers-Heijboer, D. Lindhout, G. Griffioen, A. Cats, J. Kleibeuker, L. Varesco, L. Bertario, M.L. Bisgaard, J. Mohr, and R. Fodde. 1995. Seven new mutations in $h M S H 2$, an HNPCC gene, identified by denaturing gradient gel electrophoresis. Am. I. Hum. Genet. 56: 10601066.

Wijnen, J., P. Meera Kahn, H. van der Klift, H. Vasen, F.H. Menko, F. Nagengast, A. Meijers-Heijboer, D. Lindhout, G. Griffioen, A. Cats, J. Kleibeuker, L. Varesco, L. Bertario, M.-
L. Bisgaard, J. Mohr, R. Kolodner, and R. Fodde. 1996. Majority of $h M L H 1$ mutations responsible for hereditary nonpolyposis colorectal cancer (HNPCC) cluster at the exonic region 15-16. Am. J. Hum. Genet. 58: 300-307.

Williamson, M.S., J.C. Game, and S. Fogel. 1985. Meiotic gene conversion mutants in Saccharomyces cerevisiae. I. Isolation and characterization of pms1-1 and pms1-2. Genetics 110: 609-646.

Yao, M. and Y.W. Kom. 1994. Strand-specific cleavage of mismatch-containing DNA by deoxyinosine 3 '-endonuclease from Escherichia coli. J. Biol. Chem. 269: 31390-31396.

Yeh, Y.C., H.F. Liu, C.A. Ellis, and A.-L. Lu. 1994. Mammalian topoisomerase I has base mismatch nicking activity. J. Biol. Chem. 269: 15498-15504. 


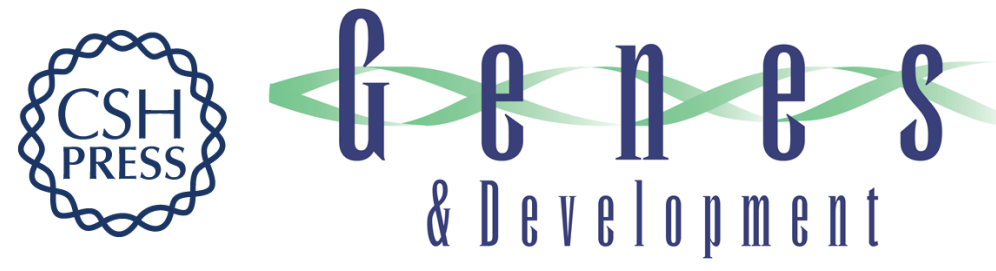

\section{Biochemistry and genetics of eukaryotic mismatch repair.}

R Kolodner

Genes Dev. 1996, 10:

Access the most recent version at doi:10.1101/gad.10.12.1433

References This article cites 84 articles, 49 of which can be accessed free at: http://genesdev.cshlp.org/content/10/12/1433.full.html\#ref-list-1

License

Email Alerting Receive free email alerts when new articles cite this article - sign up in the box at the top Service right corner of the article or click here.

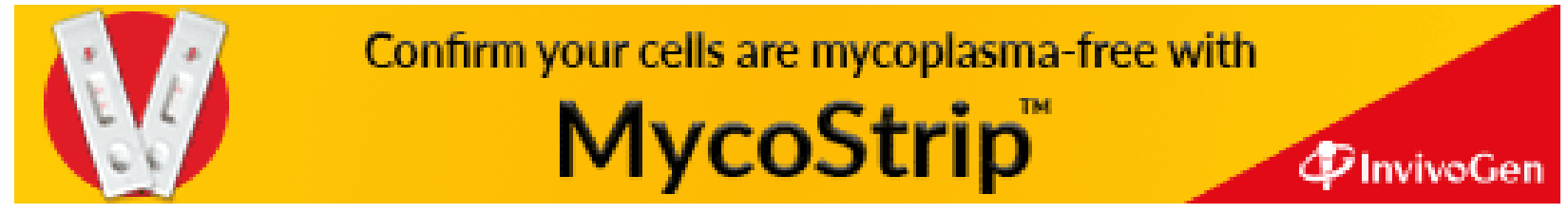

